\title{
Signature Rewriting in Gröbner Basis Computation
}

\author{
Christian Eder \\ INRIA, Paris-Rocquencourt Center, PolSys \\ Project \\ UPMC, Univ. Paris 06, LIP6 \\ CNRS, UMR 7606, LIP6 \\ UFR Ingénierie 919, LIP6 \\ Case 169, 4, Place Jussieu, F-75252 Paris \\ Christian.Eder@inria.fr
}

\author{
Bjarke Hammersholt Roune \\ Department of Mathematics \\ University of Kaiserslautern \\ Postbox 3049 \\ 67653 Kaiserslautern, Germany \\ www.broune.com
}

\begin{abstract}
We introduce the RB algorithm for Gröbner basis computation, a simpler yet equivalent algorithm to F5GEN. RB contains the original unmodified $\mathbf{F}_{\mathbf{5}}$ algorithm as a special case, so it is possible to study and understand $\mathbf{F}_{\mathbf{5}}$ by considering the simpler RB. We present simple yet complete proofs of this fact and of $\mathbf{F}_{5}$ 's termination and correctness.

$\mathbf{R B}$ is parametrized by a rewrite order and it contains many published algorithms as special cases, including SB. We prove that $\mathbf{S B}$ is the best possible instantiation of $\mathbf{R B}$ in the following sense. Let $\mathbf{X}$ be any instantiation of $\mathbf{R B}$ (such as $\mathbf{F}_{\mathbf{5}}$ ). Then the S-pairs reduced by $\mathbf{S B}$ are always a subset of the S-pairs reduced by $\mathbf{X}$ and the basis computed by $\mathbf{S B}$ is always a subset of the basis computed by $\mathbf{X}$.
\end{abstract}

\section{Categories and Subject Descriptors}

G.4 [Mathematical Software]: Algorithm design and analysis

\section{Keywords}

Gröbner basis, Syzygy Gröbner basis, Signature Gröbner basis, Signature rewriting, F5

\section{INTRODUCTION}

The $\mathbf{F}_{\mathbf{5}}$ algorithm [5] is a significant development in Gröbner basis computation and the difficulty of understanding it is a widely cited concern. We address this problem in this paper by introducing $\mathbf{R B}$, an algorithm simpler than yet equivalent to F5GEN [9]. RB contains the original unmodified $\mathbf{F}_{\mathbf{5}}$ as a special case [9], so we can understand and prove statements about $\mathbf{F}_{\mathbf{5}}$ by considering the simpler $\mathbf{R B}$. In this way we present simplified proofs of the termination and correctness of $\mathbf{F}_{\mathbf{5}}$. Much of the paper is concerned with showing exactly how to derive $\mathbf{F}_{\mathbf{5}}$ as a special case of $\mathbf{R B}$.

There are many publications that present signature-based algorithms for Gröbner basis computation. Seeing as these algorithms are based on signatures, they are all somehow related to $\mathbf{F}_{\mathbf{5}}$, yet it is often not clear what the exact relationship is - is $\mathbf{F}_{\mathbf{5}}$ a special case of the new algorithm? If not, what are the exact differences? Pan, Hu and Wang [9] (PHW) addressed this problem by introducing the F5GEN algorithm. F5GEN contains many published algorithms as special cases which makes it possible to compare algorithms theoretically. PHW pose the open problem of which instantiation of F5GEN is faster:
Moreover, with this proved F5GEN algorithm, researchers can shift their focus on the different variants of the F5GEN algorithm and find out the fastest one. — Pan, Hu, Wang [9]

We answer this open problem by proving that the SB algorithm [10] is the best possible instantiation of $\mathbf{R B}$ (and hence F5GEN) in the following sense. Let $\mathbf{X}$ be any instantiation of $\mathbf{R B}$ (such as $\mathbf{F}_{\mathbf{5}}$ ). Then the S-pairs reduced by $\mathbf{S B}$ are always a subset of the S-pairs reduced by $\mathbf{X}$ up to signature and the basis computed by $\mathbf{S B}$ is always a subset of the basis computed by $\mathbf{X}$ up to sig-lead pairs.

Roune and Stillman's algorithm SB [10] is an improvement of Gao, Volny and Wang's algorithm GVW [8]. SB is equivalent to Arri and Perry's algorithm AP [2] and also to the modification GVWHS [12] of GVW, so all statements made about $\mathbf{S B}$ also apply to AP and GVWHS.

Section 2 introduces terminology and SB. Section 3 introduces $\mathbf{R B}$ and proves that $\mathbf{S B}$ is the best possible instantiation of $\mathbf{R B}$. Section 4 introduces $\mathbf{R B}_{\mathbf{5}}$, a special case of $\mathbf{R B}$ that is more similar to $\mathbf{F}_{\mathbf{5}}$. Section 5 proves that $\mathbf{F}_{\mathbf{5}}$ is a special case of $\mathbf{R B}_{\mathbf{5}}$ (and hence $\mathbf{R B}$ ). Section 6 proves termination of $\mathbf{R B}$ (and hence $\mathbf{F}_{\mathbf{5}}$ ).

REMARK 1. The $\mathbf{F}_{\mathbf{5}}$ paper [6] requires that the input polynomials form a homogeneous regular sequence. Faugère must have known of an algorithm without these restrictions since the $\mathbf{F}_{\mathbf{5}}$ paper contains benchmarks on ideals that do not meet the restrictions. In this paper, the term $\mathbf{F}_{5}$ refers exclusively to $\mathbf{F}_{\mathbf{5}}$ exactly as described in the $\mathbf{F}_{\mathbf{5}}$ paper [6] including the restriction to homogeneous regular sequences.

\section{THE SIGNATURE BASIS ALGORITHM}

We briefly introduce $\mathbf{S B}$ in this section. We refer to [10] for a full treatment of SB with proofs. If we consider a polynomial ring $R$ then the main differences between $\mathbf{S B}$ and the classic Buchberger algorithm is that everything is lifted from $R$ to $R^{m}$, that polynomial reduction is constrained in that some reduction steps are not allowed and that the S-pair elimination criteria are more powerful. It is not actually necessary to represent polynomials in $R^{m}$ when implementing SB on a computer (see Remark 2).

\subsection{Notation and Terminology}

Let $R$ be a polynomial ring over a field $\kappa$. All polynomials $f \in R$ can be uniquely written as a finite sum $f=\sum_{c x^{v} \in M} c x^{v}$ where $c \in \kappa, x^{v}:=\prod_{i} x_{i}^{v_{i}}$ and $M$ is minimal. The elements of $M$ are the terms of $f$. A monomial 
is a polynomial with exactly one term. A monomial with a coefficient of 1 is monic. Neither monomials nor terms of polynomials are necessarily monic. We write $f \simeq g$ for $f, g \in R$ if there exists a non-zero $s \in \kappa$ such that $f=s g$.

Let $R^{m}$ be a free $R$-module and let $\boldsymbol{e}_{1}, \ldots, \boldsymbol{e}_{m}$ be the standard basis of unit vectors in $R^{m}$. All module elements $\alpha \in$ $R^{m}$ can be uniquely written as a finite $\operatorname{sum} \alpha=\sum_{a \boldsymbol{e}_{i} \in M} a \boldsymbol{e}_{i}$ where the $a$ are monomials and $M$ is minimal. The elements of $M$ are the terms of $\alpha$. A module monomial is an element of $R^{m}$ with exactly one term. A module monomial with a coefficient of 1 is monic. Neither module monomials nor terms of module elements are necessarily monic. Let $\alpha \simeq \beta$ for $\alpha, \beta \in R^{m}$ if $\alpha=s \beta$ for some non-zero $s \in \kappa$.

Let $\leq$ denote two different orders - one for $R^{m}$ and one for $R$. The order for $R$ is a monomial order, which means that it is a well-order on the set of monomials in $R$ such that $a \leq b$ implies $c a \leq c b$ for all monomials $a, b, c \in R$. The order for $R^{m}$ is a module monomial order which means that it is a well-order on the set of module monomials in $R^{m}$ such that $L \leq T$ implies $c L \leq c T$ for all module monomials $L, T \in R^{m}$ and monomials $c \in R$. We require the two orders to be compatible in the sense that $a \leq b$ if and only if $a \boldsymbol{e}_{i} \leq b \boldsymbol{e}_{i}$ for all monomials $a, b \in R$ and $i=1, \ldots, m$.

Consider a finite sequence of polynomials $g_{1}, \ldots, g_{m} \in R$ that we call the input polynomials. Define the homomorphism $\alpha \mapsto \bar{\alpha}$ from $R^{m}$ to $R$ by $\bar{\alpha}:=\sum_{i=1}^{m} \alpha_{i} g_{i} . \alpha \in R^{m}$ is a syzygy if $\bar{\alpha}=0$.

Let the lead term $\operatorname{lt}(f)$ be the $\leq$-maximal term of $f \in R \backslash$ $\{0\}$. Let the signature $\mathfrak{s}(\alpha)$ be the $\leq$-maximal term of $\alpha \in$ $R^{m} \backslash\{0\}$. In this way every non-syzygy module element $\alpha \in$ $R^{m}$ has two main associated characteristics - the signature $\mathfrak{s}(\alpha) \in R^{m}$ and the lead term $\operatorname{lt}(\bar{\alpha}) \in R$ of its image $\bar{\alpha}$ in $R$. Lead terms and signatures include a coefficient for mathematical convenience, though an implementation of SB need not store the signature coefficients. If $a \boldsymbol{e}_{i}=\mathfrak{s}(\alpha)$ then ind $(\alpha):=i$ is the index of signature of $\alpha$.

Let $\mathcal{G} \subseteq R^{m}$ be finite and assume that $\mathfrak{s}(\alpha) \simeq \mathfrak{s}(\beta) \Rightarrow$ $\alpha=\beta$ for $\alpha, \beta \in \mathcal{G} . \alpha, \beta \in R^{m}$ are equal up to sig-poly pairs if $\mathfrak{s}(\alpha)=\mathfrak{s}(s \beta)$ and $\bar{\alpha}=\overline{s \beta}$ for some non-zero $s \in \kappa$.

Consider the unique extension of the module monomial order $<$ to module monomials that may have negative exponents such that $a \boldsymbol{e}_{i}<b \boldsymbol{e}_{j} \Leftrightarrow c a \boldsymbol{e}_{i}<c b \boldsymbol{e}_{j}$ for all monomials $a, b, c \in R$. The sig-lead ratio of $\alpha \in R^{m}$ is $r_{\alpha}:=\frac{\mathfrak{s}(\alpha)}{\operatorname{lt}(\bar{\alpha})}$.

This notation is the SB notation [11], which is improved from the notation of the preceding paper [10]. In particular, there is no module $R^{n}$ and no function $\phi: R^{n} \rightarrow R^{m}$.

REMARK 2. The SB notation using module elements differs from the standard notation which considers sig-poly pairs $(\mathfrak{s}(\alpha), \bar{\alpha})$ in place of $\alpha \in R^{m}$. SB can also use sig-poly pairs (see Section 5.1). We believe that the $\mathbf{S B}$ notation makes signature algorithms easier to understand and reason about.

\subsection{Reduction With Signatures}

Both SB and the classic Buchberger algorithm are based on reducing S-pairs. We first describe classic polynomial reduction and then describe $\mathbf{S B}$ 's reduction in similar terms.

\section{Classic polynomial reduction}

Let $f \in R$ and let $t$ be a term of $f$. Then we can reduce $t$ by $g \in R$ if lt $(g) \mid t$ or equivalently if

- there exists a monomial $b$ such that $\operatorname{lt}(b g)=t$.
The outcome of the reduction step is then $f-b g$ and $g$ is the reducer. It holds that $\mathrm{lt}(b g) \leq \mathrm{lt}(f)$, but that is not listed as a requirement since it is implied. If lt $(b g) \simeq \operatorname{lt}(f)$ then the reduction step is a top reduction step and otherwise it is a tail reduction step.

The result of classic polynomial reduction of $f \in R$ is a polynomial $h \in R$ that has been calculated from $f$ through a sequence of reduction steps such that $h$ cannot be further reduced. The reduction is a tail reduction if only tail reduction steps are allowed and it is a top reduction if only top reduction steps are allowed.

The implied condition that lt $(b g) \leq \operatorname{lt}(f)$ is equivalent to lt $(f-b g) \leq \mathrm{lt}(f)$ so during classic polynomial reduction it is not allowed to increase the leading term. For tail reduction we perform only those reduction steps that do not change the leading term at all.

\section{Signature reduction}

Let $\alpha \in R^{m}$ and let $t$ be a term of $\bar{\alpha}$. Then we can $\mathfrak{s}$-reduce $t$ by $\beta \in R^{m}$ if

- there exists a monomial $b$ such that $\operatorname{lt}(\overline{b \beta})=t$ and

$$
\text { - } \mathfrak{s}(b \beta) \leq \mathfrak{s}(\alpha) \text {. }
$$

The outcome of the $\mathfrak{s}$-reduction step is then $\alpha-b \beta$ and $\beta$ is the $\mathfrak{s}$-reducer. The second condition is analogous to the implied condition lt $(b g) \leq \operatorname{lt}(f)$ from classic polynomial reduction, the condition is just lifted to $R^{m}$ so that it involves signatures. When $\beta$ s-reduces $t$ we also say for convenience that $b \beta$ s-reduces $\alpha$. That way $b$ is introduced implicitly instead of having to repeat the equation $\operatorname{lt}(\overline{b \beta})=t$.

Just as for classic polynomial reduction, if $\operatorname{lt}(\overline{b \beta}) \simeq \operatorname{lt}(\bar{\alpha})$ then the $\mathfrak{s}$-reduction step is a top $\mathfrak{s}$-reduction step and otherwise it is a tail $\mathfrak{s}$-reduction step. We need words for the analogous distinction for signatures, so if $\mathfrak{s}(b \beta) \simeq \mathfrak{s}(\alpha)$ then the reduction step is a singular $\mathfrak{s}$-reduction step and otherwise it is a regular $\mathfrak{s}$-reduction step.

The result of $\mathfrak{s}$-reduction of $\alpha \in R^{m}$ is a $\gamma \in R^{m}$ that has been calculated from $\alpha$ through a sequence of $\mathfrak{s}$-reduction steps such that $\gamma$ cannot be further $\mathfrak{s}$-reduced. The reduction is a tail $\mathfrak{s}$-reduction if only tail $\mathfrak{s}$-reduction steps are allowed and it is a top $\mathfrak{s}$-reduction if only top $\mathfrak{s}$-reduction steps are allowed. The reduction is a regular $\mathfrak{s}$-reduction if only regular $\mathfrak{s}$-reduction steps are allowed. A module element $\alpha \in R^{m}$ is $\mathfrak{s}$-reducible if it can be $\mathfrak{s}$-reduced. If $\alpha \mathfrak{s}$ reduces to $\gamma$ and $\gamma$ is a syzygy then we say that $\alpha \mathfrak{s}$-reduces to zero even if $\gamma \neq 0$.

As for classic polynomial reduction, the implied condition lt $(\overline{b \beta}) \leq \operatorname{lt}(\bar{\alpha})$ is equivalent to $\operatorname{lt}(\overline{\alpha-b \beta}) \leq \operatorname{lt}(\bar{\alpha})$, so during $\mathfrak{s}$-reduction it is not allowed to increase the leading term. For tail $\mathfrak{s}$-reduction we perform only those $\mathfrak{s}$-reduction steps that do not change the leading term at all. Analogously, the condition $\mathfrak{s}(b \beta) \leq \mathfrak{s}(\alpha)$ is equivalent to $\mathfrak{s}(\alpha-b \beta) \leq \mathfrak{s}(\alpha)$, so during $\mathfrak{s}$-reduction it is not allowed to increase the signature. For regular $\mathfrak{s}$-reduction, we perform only those $\mathfrak{s}$-reduction steps that do not change the signature at all.

Classic reduction is always with respect to a finite basis $B \subseteq R$. The reducers in classic polynomial reduction are chosen from the basis $B$. Analogously, $\mathfrak{s}$-reduction is always with respect to a finite basis $\mathcal{G} \subseteq R^{m}$. The $\mathfrak{s}$-reducers in $\mathfrak{s}$-reduction are chosen from the basis $\mathcal{G}$.

$\mathcal{G}$ is a signature Gröbner basis in signature $T$ if all $\alpha \in R^{m}$ with $\mathfrak{s}(\alpha)=T \mathfrak{s}$-reduce to zero. $\mathcal{G}$ is a signature Gröbner 
basis up to signature $T$ if $\mathcal{G}$ is a signature Gröbner basis in all signatures $L$ such that $L<T$. $\mathcal{G}$ is a signature Gröbner basis if it is a signature Gröbner basis in all signatures.

SB computes a signature Gröbner basis. If $\mathcal{G}$ is a signature Gröbner basis then $\{\bar{\alpha} \mid \alpha \in \mathcal{G}\}$ is a Gröbner basis of $\left\langle g_{1}, \ldots, g_{m}\right\rangle$, so we can use SB to compute Gröbner bases.

\subsection{S-pairs and Syzygies}

Let $f, g \in R$ and let $c:=\operatorname{gcd}(\operatorname{lt}(f), \operatorname{lt}(g))$ be the monic greatest common divisor of lt $(f)$ and lt $(g)$. In the classic Buchberger algorithm the S-polynomial between $f$ and $g$ is

$$
\frac{\operatorname{lt}(g)}{c} f-\frac{\operatorname{lt}(f)}{c} g \text {. }
$$

The classic Buchberger algorithm proceeds by reducing Spolynomials. If an S-polynomial reduces to $h \neq 0$ then $h$ is added to the basis so that the S-polynomial now reduces to zero by this larger basis. The classic Buchberger algorithm terminates once all S-polynomials between elements of the basis reduce to zero. The $\mathbf{S B}$ algorithm works the same way except that these computations are lifted from $R$ to $R^{m}$ in the following way.

Let $\alpha, \beta \in R^{m}$ and let $c:=\operatorname{gcd}(\operatorname{lt}(\bar{\alpha}), \operatorname{lt}(\bar{\beta}))$ be the monic greatest common divisor of $\operatorname{lt}(\bar{\alpha})$ and $\operatorname{lt}(\bar{\beta})$. The $S$-pair between $\alpha$ and $\beta$ is

$$
\mathcal{S}(\alpha, \beta):=\frac{\operatorname{lt}(\bar{\beta})}{c} \alpha-\frac{\operatorname{lt}(\bar{\alpha})}{c} \beta .
$$

If $\mathfrak{s}\left(\frac{\operatorname{lt}(\bar{\beta})}{c} \alpha\right) \simeq \mathfrak{s}\left(\frac{\operatorname{lt}(\bar{\alpha})}{c} \beta\right)$ then the S-pair is singular and otherwise it is regular. By "S-pair" we always mean "regular S-pair". Observe that $\mathcal{S}(\alpha, \beta) \in R^{m}$ and that $\overline{\mathcal{S}(\alpha, \beta)}$ is the S-polynomial between $\bar{\alpha}$ and $\bar{\beta}$.

SB proceeds by $\mathfrak{s}$-reducing S-pairs. If an S-pair $\mathfrak{s}$-reduces to $\gamma$ and $\bar{\gamma}$ is not zero then $\gamma$ is added to the basis. Theorem 3 implies that if all S-pairs and all $\boldsymbol{e}_{i} \mathfrak{s}$-reduce to zero then the basis is a signature Gröbner basis.

TheOrem 3. Let $T$ be a module monomial of $R^{m}$ and let $\mathcal{G} \subseteq R^{m}$ be a finite basis. Assume that all $S$-pairs $p:=$ $\mathcal{S}(\alpha, \beta)$ with $\alpha, \beta \in \mathcal{G}$ and $\mathfrak{s}(p)<T \mathfrak{s}$-reduce to zero and all $\boldsymbol{e}_{i}$ with $\boldsymbol{e}_{i}<T \mathfrak{s}$-reduce to zero. Then $\mathcal{G}$ is a signature Gröbner basis up to signature T.

The outcome of classic polynomial reduction depends on the choice of reducer, so the choice of reducer can change what the intermediate bases are in the classic Buchberger algorithm. Lemma 4 implies that all S-pairs with the same signature yield the same regular $\mathfrak{s}$-reduced result as long as we process S-pairs in order of increasing signature.

Lemma 4. Let $\alpha, \beta \in R^{m}$ and let $\mathcal{G}$ be a signature Gröbner basis up to signature $\mathfrak{s}(\alpha)=\mathfrak{s}(\beta)$. If $\alpha$ and $\beta$ are both regular top $\mathfrak{s}$-reduced then $\operatorname{lt}(\bar{\alpha})=\operatorname{lt}(\bar{\beta})$ or $\bar{\alpha}=\bar{\beta}=0$. If $\alpha$ and $\beta$ are both regular $\mathfrak{s}$-reduced then $\bar{\alpha}=\bar{\beta}$.

A signature Gröbner basis is minimal if no basis element top $\mathfrak{s}$-reduces any other basis element. Theorem 5 implies that the minimal signature Gröbner basis is unique and is contained in all signature Gröbner bases up to sig-lead pairs. SB computes a minimal signature Gröbner basis.

THEOREM 5. Let A be a minimal signature Gröbner basis and let $B$ be a signature Gröbner basis of $g_{1}, \ldots, g_{m}$. Then
SimpleSignatureBasisAlgorithm $\left(\left\{g_{1}, \ldots, g_{m}\right\} \subseteq R\right)$

$\mathcal{G} \leftarrow \emptyset$ ( $\mathcal{G}$ will be the signature Gröbner basis)

$P \leftarrow\left\{\boldsymbol{e}_{1}, \ldots, \boldsymbol{e}_{m}\right\} \quad$ ( $P$ is the set of pending reductions)

$H \leftarrow\langle 0\rangle \subseteq R^{m}$ ( $H$ will be the initial module of syzygies)

while $P \neq \emptyset$ do

$p \leftarrow$ an element of $P$ with $\leq$-minimal signature

$P \leftarrow P \backslash\{p\}$

$p^{\prime} \leftarrow$ result of regular $\mathfrak{s}$-reducing $p$

if $\overline{p^{\prime}}=0$ then

$H \leftarrow H+\left\langle\mathfrak{s}\left(p^{\prime}\right)\right\rangle$

else if $p^{\prime}$ is not singular top reducible then

$P \leftarrow P \cup\left\{\mathcal{S}\left(\alpha, p^{\prime}\right) \mid \alpha \in \mathcal{G}\right.$ and $\mathcal{S}\left(\alpha, p^{\prime}\right)$ is regular $\}$

$\mathcal{G} \leftarrow \mathcal{G} \cup\left\{p^{\prime}\right\}$

end if

end while

return $(\mathcal{G}, H)$

Figure 1: Pseudo code for a simple version of SB.

it holds for all $\alpha \in A$ that there exists a non-zero scalar $c \in \kappa$ and $a \beta \in B$ such that $\mathfrak{s}(\alpha)=c \mathfrak{s}(\beta)$ and $\operatorname{lt}(\bar{\alpha})=c \operatorname{lt}(\bar{\beta})$.

We can extract a Gröbner basis from a signature Gröbner basis, but that is not the only reason to be interested in signature Gröbner bases. It is also possible to extract a Gröbner basis of the syzygy module of the input basis $\left\{g_{1}, \ldots, g_{m}\right\}$ from a signature Gröbner basis. Note that this is the syzygy module of the input basis rather than the syzygy module of a Gröbner basis of the same ideal. The former is in general much harder to compute than the latter.

The key to computing the syzygy module is Theorem 6 which implies that we can determine the initial module of the module of syzygies from looking at those S-pairs and $\boldsymbol{e}_{i}$ that regular $\mathfrak{s}$-reduce to zero. If we are computing with full elements of $R^{m}$ (as opposed to sig-poly pairs) and we have stored the syzygies that were computed (as opposed to storing just their signatures) then those syzygies will form the minimal Gröbner basis of the syzygy module.

TheOREM 6. Let $\alpha \in R^{m}$ be a syzygy and let $\mathcal{G}$ be a signature Gröbner basis up to signature $\mathfrak{s}(\alpha)$. Then there exists a $\beta \in R^{m}$ with $\mathfrak{s}(\beta) \mid \mathfrak{s}(\alpha)$ such that $\beta$ is an $S$-pair or has the form $\boldsymbol{e}_{i}$ and such that $\beta$ regular $\mathfrak{s}$-reduces to zero.

Figure 1 contains pseudo code for a simple version of SB that returns a signature Gröbner basis $\mathcal{G}$ and and also the initial submodule $H$ of the syzygy module of $g_{1}, \ldots, g_{m}$. This pseudo code is intended to succinctly state the essence of SB without getting bogged down in any of the complexities of implementation on a computer. Among many other things, a reasonable implementation would use criteria to eliminate S-pairs (see Section 2.4) and use the sig-poly pair optimization (see Remark 2).

We stated that SB proceeds by $\mathfrak{s}$-reducing S-pairs and then adding the $\mathfrak{s}$-reduced result to the basis if it is not a syzygy. In Figure 1 we regular $\mathfrak{s}$-reduce the S-pair $p$ and then add the regular $\mathfrak{s}$-reduced result $p^{\prime}$ to the basis if it is not a syzygy and not singular $\mathfrak{s}$-reducible. The reason for this is that if $p^{\prime}$ is singular $\mathfrak{s}$-reducible then $\mathfrak{s}$-reduction of $p^{\prime}$ is going to $\mathfrak{s}$-reduce $p^{\prime}$ to zero anyway so we might as well not spend the time on doing that $\mathfrak{s}$-reduction.

\subsection{S-Pair Elimination}


Three things can happen when SB regular $\mathfrak{s}$-reduces an S-pair in signature $T$ and gets a result $\gamma \in R^{m}$.

Syzygy If $\gamma$ is a syzygy then $T$ is recorded in $H$.

Singular If $\gamma$ is singular top $\mathfrak{s}$-reducible then $\gamma \mathfrak{s}$-reduces to zero so $\gamma$ is discarded.

Basis Otherwise $\gamma$ is recorded in $\mathcal{G}$ as a new basis element.

For these three cases $T$ is respectively a syzygy, singular or basis signature. This is well defined due to Lemma 4 .

If $L$ is a syzygy signature and $L \mid T$ then $T$ is also a syzygy signature. SB eliminates an S-pair $p$ by the signature criterion if $\mathfrak{s}(p) \in H$ since then $\mathfrak{s}(p)$ is syzygy.

The Koszul syzygy between $\alpha, \beta \in \mathcal{G}$ is $\mathcal{K}(\alpha, \beta):=\bar{\beta} \alpha-$ $\bar{\alpha} \beta$. If $\mathfrak{s}(\bar{\beta} \alpha) \not{\mathfrak{s}}(\bar{\alpha} \beta)$ then the Koszul syzygy is regular. By "Koszul syzygy" we always mean "regular Koszul syzygy". SB eliminates an S-pair $p$ by the Koszul criterion if there exists a Koszul syzygy $\sigma$ such that $\mathfrak{s}(p)=\mathfrak{s}(\sigma)$. In this case $\mathfrak{s}(p)$ is recorded in $H$ since $\mathfrak{s}(p)$ is syzygy.

A signature $T$ is predictably syzygy if $\mathfrak{s}(p)=\mathfrak{s}(\sigma)$ for a Koszul syzygy $\sigma$ or if there exists a syzygy $\sigma \in R^{m}$ such that $\mathfrak{s}(\sigma)<T$ and $\mathfrak{s}(\sigma) \mid T$. The combined effect of the signature criterion and the Koszul criterion is to eliminate all S-pairs in predictably syzygy signatures.

If there are two or more S-pairs in the same signature $T$, then we only have to regular $\mathfrak{s}$-reduce one of them as they all regular $\mathfrak{s}$-reduce to the same thing by Lemma 4 . Since s-reduction proceeds by decreasing the lead term, we can speed up the process by choosing an S-pair $p$ in signature $T$ whose lead term $\operatorname{lt}(\bar{p})$ is minimal. If $\mathfrak{s}(\mathcal{S}(\alpha, \beta))=\mathfrak{s}(a \alpha)$, then we get the same result from regular $\mathfrak{s}$-reducing $\mathcal{S}(\alpha, \beta)$ as for regular $\mathfrak{s}$-reducing $a \alpha$. So we should choose the $a \alpha \in$ $M$ with minimal lead term lt $(\overline{a \alpha})$, where $M$ is the set

$\{a \alpha \mid \alpha \in \mathcal{G}, a$ is a monomial and $\mathfrak{s}(a \alpha)=T\}$.

Note that $\alpha$ might not be involved in any S-pair in signature $T$. If $a \alpha$ is not regular top $\mathfrak{s}$-reducible, then $T$ is a singular signature. In this case RB eliminates all the S-pairs in signature $T$ by the singular criterion. The effect of the singular criterion is to eliminate all S-pairs in singular signatures.

\section{THE REWRITE BASIS ALGORITHM}

In this section we introduce the $\mathbf{R B}$ algorithm, which is simpler than yet equivalent to F5GEN [9]. Our motivation for studying $\mathbf{R B}$ is that it contains many published algorithms as special cases including $\mathbf{S B}$ and $\mathbf{F}_{\mathbf{5}}$.

The difference between $\mathbf{R B}$ and $\mathbf{S B}$ is that $\mathbf{R B}$ uses the concept of rewriting instead of the concept of singular $\mathfrak{s}$ reduction. This has two main consequences. First, RB uses a different criterion for eliminating S-pairs. Second, RB adds a regular $\mathfrak{s}$-reduced $\alpha \in R^{m}$ to the basis even if $\alpha$ is singular top $\mathfrak{s}$-reducible. SB does not add such $\alpha$ to the basis because such $\alpha$ s-reduce to zero anyway. RB must add such $\alpha$ to the basis because rewriting requires it.

$\mathbf{R B}$ is parametrized by a rewrite order. Despite the differences between RB and SB, PHW [9] show that GVWHS (and hence SB) is an instantiation of F5GEN (and hence $\mathbf{R B}$ ). We simplify their proof in the setting of $\mathbf{S B}$ and $\mathbf{R B}$ and extend the result to say that $\mathbf{S B}$ is the best possible instantiation of $\mathbf{R B}$ in the sense of Theorem 13 .

Figure 2 contains pseudo code for a simple version of $\mathbf{R B}$.
SimpleRewriteBasisAlgorithm $\left(\left\{g_{1}, \ldots, g_{m}\right\} \subseteq R\right)$

$\mathcal{G} \leftarrow \emptyset$ ( $\mathcal{G}$ will be the rewrite basis)

$P \leftarrow\left\{\boldsymbol{e}_{1}, \ldots, \boldsymbol{e}_{m}\right\} \quad$ ( $P$ is the set of pending reductions)

$H \leftarrow\langle 0\rangle \subseteq R^{m}$ ( $H$ will be the initial module of syzygies)

while $P \neq \emptyset$ do

$p \leftarrow$ an element of $P$ with $\leq$-minimal signature

$P \leftarrow P \backslash\{p\}$

if not RBEliminated $(p)$ then

$p^{\prime} \leftarrow$ result of regular $\mathfrak{s}$-reducing $p$

if $\overline{p^{\prime}}=0$ then

$H \leftarrow H+\left\langle\mathfrak{s}\left(p^{\prime}\right)\right\rangle$

else

$P \leftarrow P \cup\left\{\mathcal{S}\left(\alpha, p^{\prime}\right) \mid \alpha \in \mathcal{G}\right.$ and $\mathcal{S}\left(\alpha, p^{\prime}\right)$ is regular $\}$

$\mathcal{G} \leftarrow \mathcal{G} \cup\left\{p^{\prime}\right\}$ (insert $p^{\prime}$ into the basis even when $p^{\prime}$

is singular top $\mathfrak{s}$-reducible)

end if

end if

end while

return $(\mathcal{G}, H)$

RBEliminated(S-pair $a \alpha-b \beta \in R^{m}$ )

if $a \alpha$ is rewritable or $a \alpha$ is predictably syzygy then

return true (eliminate S-pair)

end if

if $b \beta$ is rewritable or $b \beta$ is predictably syzygy then

return true (eliminate $\mathrm{S}$-pair)

end if

return false (do not eliminate S-pair)

Figure 2: Pseudo code for a simple version of RB.

\subsection{Rewriting and S-pair Elimination}

Let $\preceq$ be a rewrite order, which means that $\preceq$ is a total order on $\mathcal{G}$ such that $\mathfrak{s}(\alpha) \mid \mathfrak{s}(\beta) \Rightarrow \alpha \preceq \beta$. Such an order always exists due to our assumption that $\mathfrak{s}(\alpha) \simeq \mathfrak{s}(\beta) \Rightarrow$ $\alpha=\beta$. A basis element $\alpha \in \mathcal{G}$ is a rewriter in signature $T$ if $\mathfrak{s}(\alpha) \mid T$. If $\mathfrak{s}(a \alpha)=T$ for a monomial $a$ we also say for convenience that $a \alpha$ is a rewriter of $T$. The $\preceq$-maximal rewriter in signature $T$ is the canonical rewriter. A basis element multiple $a \alpha$ is rewritable if $\alpha$ is not the canonical rewriter of $\mathfrak{s}(a \alpha)$. An S-pair $a \alpha-b \beta$ is eliminated by $\mathbf{R B}$ if $a \alpha$ is predictably syzygy or rewritable, or if $b \beta$ is predictably syzygy or rewritable.

Note that RB's S-pair elimination criterion applies equally to both components $a \alpha$ and $b \beta$ of an S-pair $a \alpha-b \beta$. No criterion in SB can eliminate an S-pair based on $\mathfrak{s}(b \beta)$ where $\mathfrak{s}(a \alpha)>\mathfrak{s}(b \beta)$. Can RB eliminate some S-pairs that RB cannot due to this difference? Since both $\mathbf{S B}$ and $\mathbf{R B}$ regular $\mathfrak{s}$-reduce at most one $\mathrm{S}$-pair in any given signature, what matters is the ability to eliminate all S-pairs in a signature. We will prove that SB has a stronger S-pair elimination criterion than $\mathbf{R B}$ does in the sense that if $\mathbf{S B}$ must regular $\mathfrak{s}$-reduce an S-pair in a signature then so must RB.

\subsection{Rewrite bases}

$\mathcal{G}$ is a rewrite basis in signature $T$ if the canonical rewriter in signature $T$ is not regular top $\mathfrak{s - r e d u c i b l e ~ o r ~ i f ~} T$ is a syzygy signature. $\mathcal{G}$ is a rewrite basis up to signature $T$ if $\mathcal{G}$ is a rewrite basis for all signatures $L$ such that $L<T$. $\mathcal{G}$ is a rewrite basis if it is a rewrite basis in all signatures. We prove Theorem 7 later in this section.

TheOREM 7. RB computes a rewrite basis. 
LEMma 8. If $\mathcal{G}$ is a rewrite basis up to signature $T$ then $\mathcal{G}$ is also a signature Gröbner basis up to signature $T$.

Proof. Suppose to get a contradiction that $\mathcal{G}$ is a rewrite basis up to signature $T$ but that it is not a signature Gröbner basis up to signature $T$. Since the monomial order is a well-order there exists an $\alpha \in R^{m}$ with minimal signature $\mathfrak{s}(\alpha)<T$ such that $\alpha$ does not $\mathfrak{s}$-reduce to zero. Then $\mathcal{G}$ is a signature basis up to signature $\mathfrak{s}(\alpha)$ and it is a rewrite basis in signature $\mathfrak{s}(\alpha)$.

Let $\beta$ be the result of regular $\mathfrak{s}$-reducing $\alpha$ and let $c \gamma$ be the canonical rewriter in signature $\mathfrak{s}(\alpha)$. Then $\mathfrak{s}(c \gamma)=$ $\mathfrak{s}(\beta)$ and both $c \gamma$ and $\beta$ are not regular top $\mathfrak{s}$-reducible, so $\operatorname{lt}(\overline{c \gamma})=\operatorname{lt}(\bar{\beta})$ by Lemma 4 . Perform the singular $\mathfrak{s}-$ reduction step $\beta-c \gamma$. Since $\mathfrak{s}(\beta-c \gamma)<\mathfrak{s}(\alpha)$ and $\mathcal{G}$ is a signature Gröbner basis up to signature $\mathfrak{s}(\alpha)$ we then get that $\beta-c \gamma \mathfrak{s}$-reduces to zero. Hence $\alpha \mathfrak{s}$-reduces to zero which is a contradiction.

SB computes a minimal signature Gröbner basis so the SB basis is a subset of the RB basis up to sig-poly pairs. Both $\mathbf{S B}$ and $\mathbf{R B}$ have to perform a regular $\mathfrak{s}$-reduction in all syzygy signatures that are not predictably syzygy, so there are no differences between the two algorithms in terms of how many S-pairs are reduced to zero. For non-syzygy signatures $T$, both algorithms will add an element to the basis with signature $T$ if and only if they reduce an S-pair in signature $T{ }^{1}$ This implies that if $\mathbf{S B}$ regular $\mathfrak{s}$-reduces an S-pair in signature $T$ then so does RB. In other words, the Spairs reduced by $\mathbf{S B}$ form a subset of the S-pairs reduced by $\mathbf{R B}$ up to signature. If $A$ is the basis computed by $\mathbf{S B}$ and $B$ is the basis computed by $\mathbf{R B}$, then $\mathbf{R B}$ regular $\mathfrak{s}$-reduced $|B|-|A| \geq 0$ more S-pairs than SB did. If $|A|=|B|$ then SB and RB reduced the same S-pairs up to signature.

Our proof of Theorem 7 is based on the following series of lemmas. Lemma 10 connects S-pairs to rewrite bases. Lemma 11 is an important technical lemma that we use here to construct S-pairs and which we use again in Section 4.1. Lemma 12 gives a precise criterion for when RB regular $\mathfrak{s - r e d u c e s}$ an $\mathrm{S}$-pair in a signature.

Lemma 9. Let $\mathcal{G}$ be a rewrite basis up to signature $T$. Let $a \alpha$ be the canonical rewriter in signature $T$ and let $b \beta$ be $a$ regular top $\mathfrak{s}$-reducer of $a \alpha$. Then $\mathcal{S}(\alpha, \beta)=a \alpha-b \beta$ and $\mathfrak{s}(\mathcal{S}(\alpha, \beta))=T$.

Proof. If $g:=\operatorname{gcd}(a, b)$ then $a \alpha-b \beta=g \mathcal{S}(\alpha, \beta)$. Suppose to get a contradiction that $g \neq 1$. Let $c \gamma$ be the canonical rewriter in signature $\frac{T}{g}$. Then $\gamma \succeq \alpha$ since $\frac{a}{g} \alpha$ is a rewriter in signature $\frac{T}{g}$. Also $\gamma \preceq \alpha$ since $g c \gamma$ is a rewriter in signature $T$. Hence $\alpha=\gamma$. This is a contradiction since then $\frac{b}{g} \beta$ is a regular top $\mathfrak{s - r e d u c e r}$ of $\frac{a}{g} \alpha=c \gamma$ but $c \gamma$ is not regular top s-reducible.

LEMMA 10. $\mathcal{G}$ is a rewrite basis up to signature $T$ if $\mathcal{G}$ is a rewrite basis in all signatures $\mathfrak{s}(p)<T$ where $p$ is an $S$-pair or $p=\boldsymbol{e}_{i}$.

Proof. Suppose to get a contradiction that $\mathcal{G}$ is not a rewrite basis up to signature $T$. Since the module monomial order $<$ is a well-order there exists a minimal non-syzygy

${ }^{1}$ If an S-pair $p$ regular $\mathfrak{s}$-reduces to $p^{\prime}$ then it is true that $\mathbf{S B}$ will not add $p^{\prime}$ to the basis if $p^{\prime}$ is singular top $\mathfrak{s}$-reducible. However, the singular criterion always eliminates such Spairs $p$ before the regular $\mathfrak{s}$-reduction happens. signature $L<T$ such that $\mathcal{G}$ is not a rewrite basis in signature $L$. Then $\mathcal{G}$ is a rewrite basis up to signature $L$. Then there exists an S-pair with signature $L$ by Lemma 9 so $\mathcal{G}$ is a rewrite basis in signature $L$ which is a contradiction.

Lemma 11. Let $\alpha \in R^{m}$, let $\mathcal{G}$ be a rewrite basis up to signature $\mathfrak{s}(\alpha)$ and let $t$ be a regular $\mathfrak{s}$-reducible term of $\bar{\alpha}$. Let $M$ be the set of $c \gamma$ that regular $\mathfrak{s}$-reduce $t$. Let $b \beta$ be the canonical rewriter in signature $L:=\min _{c \gamma \in M} \mathfrak{s}(c \gamma)$. Then

- $b \beta$ is a regular $\mathfrak{s}$-reducer of $t$,

- $b \beta$ is not regular top $\mathfrak{s}$-reducible,

- $b \beta$ is not rewritable and

- $\mathfrak{s}(b \beta)$ is not syzygy.

Proof. $b \beta$ is the canonical rewriter in signature $L<\mathfrak{s}(\alpha)$ and $\mathcal{G}$ is a rewrite basis up to signature $\mathfrak{s}(\alpha)$, which establishes the two middle statements.

$\boldsymbol{b} \boldsymbol{\beta} \in M$ : Let $c \gamma \in M$ such that $\mathfrak{s}(c \gamma)=L$. Suppose to get a contradiction that $d \delta$ regular top $\mathfrak{s}$-reduces $c \gamma$. Then $\operatorname{lt}(\overline{d \delta})=\operatorname{lt}(\overline{c \gamma})=t$ and $\mathfrak{s}(d \delta)<\mathfrak{s}(c \gamma)<\mathfrak{s}(\alpha)$, so $d \delta \in M$ and $\mathfrak{s}(d \delta)<L$ which is a contradiction. Then $\mathrm{lt}(\overline{b \beta})=$ lt $(\overline{c \gamma})=t$ by Lemma 4 so $b \beta \in M$.

$\mathfrak{s}(\boldsymbol{b} \boldsymbol{\beta})$ is not syzygy: Suppose to get a contradiction that there exists a syzygy $\sigma$ such that $\mathfrak{s}(\sigma)=\mathfrak{s}(b \beta)$. Since $\mathfrak{s}(b \beta-\sigma)<\mathfrak{s}(\alpha)$ there exists a top $\mathfrak{s}$-reducer $c \gamma$ of $b \beta-\sigma$. Then $\mathfrak{s}(c \gamma) \leq \mathfrak{s}(b \beta-\sigma)<\mathfrak{s}(b \beta)$ so $c \gamma$ is a regular top $\mathfrak{s}$-reducer of $b \beta$ but $b \beta$ is not regular top $\mathfrak{s}$-reducible.

Lemma 12. Let $\mathcal{G}$ be a rewrite basis up to signature $T$. Let $a \alpha$ be the canonical rewriter in signature $T$. Then $\mathbf{R B}$ $\mathfrak{s}$-reduces an $S$-pair in signature $T$ if and only if a $\alpha$ is regular top $\mathfrak{s}$-reducible and $T$ is not predictably syzygy.

ProOF. if: Let $b \beta$ be the regular top $\mathfrak{s}$-reducer of $a \alpha$ from Lemma 11 so that $b \beta$ is not rewritable and $\mathfrak{s}(b \beta)$ is not predictably syzygy. Then $\mathcal{S}(\alpha, \beta)=a \alpha-b \beta$ by Lemma 9 and this S-pair is not eliminated by $\mathbf{R B}$.

only if: Let $\mathcal{S}(\alpha, \beta)=a \alpha-b \beta$ such that $T=\mathfrak{s}(a \alpha)>$ $\mathfrak{s}(b \beta)$ and such that RB does not eliminate $\mathcal{S}(\alpha, \beta)$. The latter implies that $a \alpha$ is the canonical rewriter in signature $T$ and that $T$ is not predictably syzygy. Observe that $a \alpha$ is regular top $\mathfrak{s}$-reducible since $b \beta$ regular top $\mathfrak{s}$-reduces it.

Proof of Theorem 7. Suppose that RB has terminated with the basis $\mathcal{G}$. Suppose to get a contradiction that $p$ is an S-pair such that $\mathcal{G}$ is not a rewrite basis in signature $\mathfrak{s}(p)$. Let $a \alpha$ be the canonical rewriter in signature $\mathfrak{s}(p)$. Then $a \alpha$ is regular top $\mathfrak{s}$-reducible and $\mathfrak{s}(a \alpha)=\mathfrak{s}(p)$ is not syzygy, so RB has reduced an S-pair in signature $\mathfrak{s}(p)$ by Lemma 12. Then RB has also added a basis element $\beta$ with signature $\mathfrak{s}(p)$ whereby $\beta=a \alpha$ is the canonical reducer in signature $\mathfrak{s}(p)$. This is a contradiction since $\beta$ is not regular top $\mathfrak{s}$-reducible. Then $\mathcal{G}$ is a rewrite basis by Lemma 10 .

\section{3 $\mathrm{SB}$ is the Best Possible Instantiation of $\mathrm{RB}$}

Let the sig-lead ratio order $\preceq_{r}$ be the order on $\mathcal{G}$ such that $\alpha \preceq_{r} \beta$ if $r_{\alpha} \leq r_{\beta}$ or if $r_{\alpha}=r_{\beta}$ and $\mathfrak{s}(\alpha) \leq \mathfrak{s}(\beta)$.

Theorem 13. RB is equivalent to $\mathbf{S B}$ when using the sig-lead ratio rewrite order. If $\mathbf{R B}$ uses any other rewrite order, then $\mathbf{S B}$ computes a basis that is a subset of the basis computed by RB up to sig-poly pairs and $\mathbf{S B}$ regular $\mathfrak{s}$-reduces a subset of the $S$-pairs regular $\mathfrak{s}$-reduced by $\mathbf{R B}$ up to signature. 
Proof. Let RB use $\preceq_{r}$ for rewriting and let $\mathcal{G}$ be the minimal signature Gröbner basis. We will prove that $\preceq_{r}$ is a rewrite order and that $\mathcal{G}$ is a rewrite basis. Then $\mathbf{R B}$ computes $\mathcal{G}$ as it only regular $\mathfrak{s}$-reduces an S-pair in a non-syzygy signature $T$ if the basis is not already a rewrite basis in signature $T$. Thus $\mathbf{S B}$ and $\mathbf{R B}$ compute the same basis and then the result follows from the statements in Section 3.2.

$\preceq_{r}$ is a rewrite order: If $\mathfrak{s}(\alpha) \mid \mathfrak{s}(\beta)$ then $r_{\alpha} \leq r_{\beta}$ by Lemma 14 and $\mathfrak{s}(\alpha) \leq \mathfrak{s}(\beta)$ so $\alpha \preceq_{r} \beta$.

$\mathcal{G}$ is a rewrite basis: Let $a \alpha$ be a canonical rewriter. We need to prove that $a \alpha$ is not regular top $\mathfrak{s}$-reducible. Let $\gamma$ be the result of regular $\mathfrak{s}$-reducing $a \alpha$. $\mathcal{G}$ is a signature Gröbner basis, so there exists a $b \beta$ that singular top s-reduces $\gamma$. Then $r_{\alpha} \leq r_{\beta}$ by Lemma 14 so $\alpha=\beta$.

LEMMA 14. Let $\mathcal{G}$ be a signature Gröbner basis up to signature $\mathfrak{s}(a \alpha)=\mathfrak{s}(b \beta)$ where $\alpha, \beta \in \mathcal{G}$ and $a, b$ are monomials. If $b \beta$ is not regular top $\mathfrak{s}$-reducible then $r_{\alpha} \leq r_{\beta}$.

Proof. Let $\gamma$ be the result of regular $\mathfrak{s}$-reducing $a \alpha . b \beta$ and $\gamma$ are not regular top $\mathfrak{s}$-reducible so $\operatorname{lt}(\overline{a \alpha}) \geq \operatorname{lt}(\bar{\gamma})=$ lt $(\overline{b \beta})$ by Lemma 4 . Also $\mathfrak{s}(\gamma)=\mathfrak{s}(a \alpha)=\mathfrak{s}(b \beta)$, so

$$
r_{\alpha}=\frac{\mathfrak{s}(a \alpha)}{\operatorname{lt}(\overline{a \alpha})} \leq \frac{\mathfrak{s}(\gamma)}{\operatorname{lt}(\bar{\gamma})}=\frac{\mathfrak{s}(b \beta)}{\operatorname{lt}(\overline{b \beta})}=r_{\beta}
$$

\section{4. $\mathrm{RB}_{5}-$ BRINGING RB CLOSER TO $\mathrm{F}_{5}$}

In this section we define $\mathbf{R B}_{\mathbf{5}}$, which is a special case of $\mathbf{R B}$. $\mathbf{R B}_{\mathbf{5}}$ is more similar to $\mathbf{F}_{\mathbf{5}}$ than $\mathbf{R B}$ is. We will show why the differences between $\mathbf{R B}_{\mathbf{5}}$ and $\mathbf{R B}$ are not significant, except for the fact that $\mathbf{R B}$ is more general. In Section 5 we resolve the differences between $\mathbf{R B}_{\mathbf{5}}$ and $\mathbf{F}_{\mathbf{5}}$, which completes our proof that $\mathbf{F}_{\mathbf{5}}$ is a special case of $\mathbf{R B}$.

$\mathbf{R B}_{\mathbf{5}}$ is specialized from $\mathbf{R B}$ in three main ways. First, the module monomial order must be position-first, which means that if $i<j$ then $a \boldsymbol{e}_{i}<b \boldsymbol{e}_{j}$ for all monomials $a, b \in$ $R$. Second, the input basis elements $g_{1}, \ldots, g_{m}$ must form a regular sequence. Third, the rewrite order must be the $\mathbf{F}_{\mathbf{5}}$ rewrite order $\preceq_{5}$. Let $a \boldsymbol{e}_{i}:=\mathfrak{s}(\alpha)$ and $\mathfrak{s}\left(b \boldsymbol{e}_{j}\right):=\beta$. Then $\alpha \preceq_{5} \beta$ if $i<j$ or if $i=j$ and the total degree of $a$ is strictly smaller than the total degree of $b$. Break ties arbitrarily.

\subsection{Reduction in $\mathrm{RB}_{5}$}

Let $\alpha \in R^{m}$ and let $t$ be a term of $\bar{\alpha}$. We can $\mathfrak{r}$-reduce $t$ by a basis element $\beta \in \mathcal{G}$ if

- there exists a monomial $b$ such that $\operatorname{lt}(\overline{b \beta})=t$,

- $\mathfrak{s}(b \beta)<\mathfrak{s}(\alpha)$,

- $\mathfrak{s}(b \beta)$ is not syzygy and

- $b \beta$ is not rewritable.

The outcome of the $\mathfrak{r}$-reduction step is then $\alpha-b \beta$ and $\beta$ is the $\mathfrak{r}$-reducer. When $\beta \mathfrak{r}$-reduces a term of $\bar{\alpha}$ we also say for convenience that $b \beta \mathfrak{r}$-reduces $\alpha$. If $t=\operatorname{lt}(\bar{\alpha})$ then the $\mathfrak{r}$-reduction step is a top $\mathfrak{r}$-reduction step.

The first condition on $\mathfrak{r}$-reducers is the same as for $\mathfrak{s}$ reduction. The second condition requires $<$ where $\mathfrak{s}$-reduction requires only $\leq$, so $\mathfrak{r}$-reduction is implicitly regular. The final two conditions are not used by RB. In Section 5.3 we motivate these extra conditions in terms of S-pair elimination. All $\mathfrak{r}$-reducers are regular $\mathfrak{s}$-reducers but not vice versa.
Lemma 15. Let $\alpha \in R^{m}$, let $t$ be a term of $\bar{\alpha}$ and let $\mathcal{G}$ be a rewrite basis up to signature $\mathfrak{s}(\alpha)$. Then $t$ is regular $\mathfrak{s}$-reducible if and only if $t$ is $\mathfrak{r}$-reducible

Proof. If $t$ is regular $\mathfrak{s}$-reducible then by Lemma 11 there exists a regular $\mathfrak{s}$-reducer $b \beta$ of $t$ such that $\mathfrak{s}(b \beta)$ is not syzygy and $b \beta$ is not rewritable. So $b \beta$ is an $\mathfrak{r}$-reducer.

During regular $\mathfrak{s}$-reduction there can be a choice of which regular $\mathfrak{s}$-reducer to use. Lemma 15 shows that the effect of the extra conditions imposed on $\mathfrak{r}$-reducers is to exclude some of the $\mathfrak{s}$-reducers from consideration, but it is never the case that all of the $\mathfrak{s}$-reducers are excluded. The outcome of regular $\mathfrak{s}$-reduction does not depend on the choice of $\mathfrak{s}$ reducer up to sig-poly pairs, which proves Corollary 16.

Corollary 16. Let $\alpha \in R^{m}$ and let $\mathcal{G}$ be a rewrite basis up to signature $\mathfrak{s}(\alpha)$. Let $\alpha_{\mathfrak{s}}$ be the result of regular $\mathfrak{s}$-reducing $\alpha$ and let $\alpha_{\mathfrak{r}}$ be the result of $\mathfrak{r}$-reducing $\alpha$. Then $\mathfrak{s}\left(\alpha_{\mathfrak{s}}\right)=\mathfrak{s}\left(\alpha_{\mathfrak{r}}\right)$ and $\overline{\alpha_{\mathfrak{s}}}=\overline{\alpha_{\mathfrak{r}}}$.

\subsection{S-pair elimination in $\mathrm{RB}_{5}$}

Due to the extra assumptions on the input, $\mathbf{R B}_{\mathbf{5}}$ can eliminate all S-pairs that reduce to zero by considering only the Koszul syzygies. This is stated already in the $\mathbf{F}_{\mathbf{5}}$ paper [6]. RB uses condition (2) of Theorem 17 while $\mathbf{R B}_{\mathbf{5}}$ uses condition (4). Theorem 17 implies that these two are equivalent.

THEOREM 17. Let $\mathcal{G}$ be a signature Gröbner basis up to signature $\boldsymbol{e}_{i}$. Assume that the input basis element $g_{i}$ is not a zero divisor of $R /\left\langle g_{1}, \ldots, g_{i-1}\right\rangle$. Assume that the module monomial order $<$ on $R^{m}$ has the property that be $\boldsymbol{e}_{j}<a \boldsymbol{e}_{i}<$ $c e_{k}$ for all $j, k$ such that $j<i<k$ and for all monomials $a, b, c \in R$. Then the following statements are equivalent for all monomials $a \in R$.

\section{The signature a $\boldsymbol{e}_{i}$ is syzygy.}

2. The signature a $\boldsymbol{e}_{i}$ is predictably syzygy.

3. $\exists \alpha \in \mathcal{G}:$ ind $(\alpha)<i$ and $\mathfrak{s}\left(\mathcal{K}\left(\boldsymbol{e}_{i}, \alpha\right)\right) \mid a \boldsymbol{e}_{i}$.

4. $\exists \alpha \in \mathcal{G}$ : ind $(\alpha)<i$ and $\operatorname{lt}(\bar{\alpha}) \mid a$.

Proof. Let $G:=\{\alpha \in \mathcal{G} \mid \operatorname{ind}(\alpha)<i\}, \bar{G}:=\{\bar{\alpha} \mid \alpha \in G\}$ and $F:=\left\langle g_{1}, \ldots, g_{i-1}\right\rangle$. That $(3) \Rightarrow(2) \Rightarrow(1)$ is immediate.

$\overline{\boldsymbol{G}}$ is a Gröbner basis of $\boldsymbol{F}$ : If $\alpha \in G$ then $\alpha_{j}=0$ for $j \geq i$ so it is immediate that $\langle\bar{G}\rangle \subseteq\left\langle g_{1}, \ldots, g_{i-1}\right\rangle$. To prove the other inclusion, let $f \in F$. We need to prove that $f$ reduces to zero on classic polynomial reduction by $\bar{G}$.

There exists an $\alpha \in R^{m}$ such that $f=\bar{\alpha}$ and $\alpha_{j}=0$ for $j \geq i$. Then ind $(\alpha)<i$ so $\mathfrak{s}(\alpha)<\boldsymbol{e}_{i}$ whereby $\alpha \mathfrak{s}$-reduces to zero when $\mathfrak{s}$-reducing by $\mathcal{G}$. All the $\mathfrak{s}$-reducers $\gamma \in \mathcal{G}$ in that $\mathfrak{s}$-reduction must have $\mathfrak{s}(\gamma) \leq \mathfrak{s}(\alpha)$ whereby ind $(\gamma)<i$. Thus $\alpha$ also $\mathfrak{s}$-reduces to zero when $\mathfrak{s}$-reducing by $G$. Then $\bar{\alpha}=f$ reduces to zero on classic polynomial reduction by $\bar{G}$.

$(1) \Rightarrow(4)$ : Let $\beta \in R^{m}$ be a syzygy such that $\mathfrak{s}(\beta)=a \boldsymbol{e}_{i}$. Then $\beta_{j}=0$ for all $j>i$ whereby $\beta_{i} g_{i} \in F$ since

$$
0=\bar{\beta}=\sum_{j=1}^{m} \beta_{j} g_{j}=\beta_{i} g_{i}+\sum_{j=1}^{i-1} \beta_{j} g_{j} .
$$

Hence $\beta_{i} \in F$ as $g_{i}$ is not a zero divisor. As $\bar{G}$ is a Gröbner basis of $F$ there exists an $\alpha \in G$ such that $\operatorname{lt}(\bar{\alpha}) \mid \operatorname{lt}\left(\beta_{i}\right)=a$.

$(4) \Rightarrow(3)$ : Let $\alpha \in \mathcal{G}$ such that ind $(\alpha)<i$ and $\operatorname{lt}(\bar{\alpha}) \mid a$. By definition $\mathcal{K}\left(\boldsymbol{e}_{i}, \alpha\right)=\bar{\alpha} \boldsymbol{e}_{i}-g_{i} \alpha$. As lt $(\bar{\alpha}) \boldsymbol{e}_{i}>\operatorname{lt}\left(g_{i}\right) \mathfrak{s}(\alpha)$ we see that $\mathfrak{s}\left(\mathcal{K}\left(\boldsymbol{e}_{i}, \alpha\right)\right)=\operatorname{lt}(\bar{\alpha}) \boldsymbol{e}_{i} \mid a \boldsymbol{e}_{i}$. 


\begin{tabular}{rrrr}
$\phi_{i} \in \mathcal{G}$ & reduced from & lt $\left(\overline{\phi_{i}}\right)$ & $\mathfrak{s}\left(\phi_{i}\right)$ \\
\hline$\phi_{1}$ & $\boldsymbol{e}_{1}$ & $y^{3}$ & $\boldsymbol{e}_{1}$ \\
$\phi_{2}$ & $\boldsymbol{e}_{2}$ & $x y z$ & $\boldsymbol{e}_{2}$ \\
$\phi_{3}$ & $y^{2} \phi_{2}-x z \phi_{1}=\mathcal{S}\left(\phi_{2}, \phi_{1}\right)$ & $x^{3} z^{2}$ & $y^{2} \boldsymbol{e}_{2}$ \\
$\phi_{4}$ & $\boldsymbol{e}_{3}$ & $y z^{2}$ & $\boldsymbol{e}_{3}$ \\
$\phi_{5}$ & $x \phi_{3}-z \phi_{2}=\mathcal{S}\left(\phi_{3}, \phi_{2}\right)$ & $x z^{3}$ & $x \boldsymbol{e}_{3}$ \\
$\phi_{6}$ & $y^{2} \phi_{3}-z^{2} \phi_{1}=\mathcal{S}\left(\phi_{3}, \phi_{1}\right)$ & $x^{2} z^{3}$ & $y^{2} \boldsymbol{e}_{3}$ \\
$\phi_{7}$ & $y \phi_{5}-z^{2} \phi_{2}=\mathcal{S}\left(\phi_{5}, \phi_{2}\right)$ & $x^{2} y^{2} t$ & $x y \boldsymbol{e}_{3}$ \\
$\phi_{8}$ & $x \phi_{5}-\phi_{6}=\mathcal{S}\left(\phi_{5}, \phi_{6}\right)$ & $z^{5}$ & $x^{2} \boldsymbol{e}_{3}$ \\
$\phi_{9}$ & $x \phi_{6}-z \phi_{3}=\mathcal{S}\left(\phi_{6}, \phi_{3}\right)$ & $x^{4} z t$ & $x y^{2} \boldsymbol{e}_{3}$ \\
$\phi_{10}$ & $y \phi_{8}-z^{3} \phi_{4}=\mathcal{S}\left(\phi_{8}, \phi_{4}\right)$ & $x^{3} y^{2} t$ & $x^{2} y \boldsymbol{e}_{3}$ \\
$\phi_{11}$ & $x^{3} \phi_{4}-y \phi_{3}=\mathcal{S}\left(\phi_{4}, \phi_{3}\right)$ & $x^{4} y t$ & $x^{3} \boldsymbol{e}_{3}$ \\
$\phi_{12}$ & $z \phi_{11}-x^{3} \phi_{2}=\mathcal{S}\left(\phi_{11}, \phi_{2}\right)$ & $x^{3} z t^{3}$ & $x^{3} z \boldsymbol{e}_{3}$ \\
$\phi_{13}$ & $y \phi_{10}-x^{3} \phi_{1}=\mathcal{S}\left(\phi_{10}, \phi_{1}\right)$ & $x^{5} z t$ & $x^{2} y^{2} \boldsymbol{e}_{3}$ \\
$\phi_{14}$ & $x \phi_{12}-\phi_{9}=\mathcal{S}\left(\phi_{12}, \phi_{9}\right)$ & $x^{4} t^{4}$ & $x^{4} z \boldsymbol{e}_{3}$
\end{tabular}

Figure 3: Computations for $\mathrm{RB}_{5}$ in Example 19.

\subsection{Examples}

Example 18 shows a homogeneous ideal where $\mathbf{R B}_{\mathbf{5}}$ (and hence $\mathbf{F}_{\mathbf{5}}$ ) computes a larger basis than $\mathbf{S B}$ does. Example 19 shows details of how $\mathbf{R B}_{\mathbf{5}}$ computes a basis.

ExAmPle 18. Consider the homogenized Eco-6 ideal using the graded reverse lexicographic monomial order. Here $\mathbf{R B}_{5}$ computes a basis with 100 elements while $\mathbf{S B}$ computes a basis with 87 elements. Both $\mathbf{R B}_{5}$ and $\mathbf{S B}$ compute a basis element $\alpha$ with $\mathfrak{s}(\alpha)=x_{0} x_{2} \boldsymbol{e}_{5} . \mathbf{R B}_{5}$ additionally computes basis elements $\beta$ and $\gamma$ with $\mathfrak{s}(\beta)=x_{0} x_{2} x_{3}^{2} \boldsymbol{e}_{5}$ and $\mathfrak{s}(\gamma)=x_{0} x_{2} x_{3} x_{4} \boldsymbol{e}_{5}$. As $\beta$ and $\gamma$ are not necessary to have a signature Gröbner basis, we can replacing them with a single element with signature $x_{0} x_{2} x_{3} \boldsymbol{e}_{5}$ and thus get a rewrite basis that contains fewer elements than the basis computed by $\mathbf{R B}_{\mathbf{5}}$. Furthermore, the basis computed by $\mathbf{S B}$ is not a rewrite basis with respect to the $\mathbf{F}_{\mathbf{5}}$ rewrite order.

EXAMPLE 19. Let $\kappa$ be the finite field with 13 elements and let $R:=\kappa[x, y, z, t]$. Let $<$ be the graded reverse lexicographic monomial order. Consider the three input elements

$$
\begin{aligned}
& g_{1}:=-2 y^{3}-x^{2} z-2 x^{2} t-3 y^{2} t, \quad g_{2}:=3 x y z+2 x y t, \\
& g_{3}:=2 x y z-2 y z^{2}+2 z^{3}+4 y z t .
\end{aligned}
$$

Figure 3 shows the $\mathfrak{r}$-reductions performed by $\mathbf{R B}_{\mathbf{5}}$ to compute the rewrite basis $\left\{\phi_{1}, \ldots, \phi_{14}\right\}$. $\mathbf{S B}$ regular $\mathfrak{s}$-reduce the same $S$-pairs except for the ones that lead to $\phi_{10}$ and $\phi_{13}$, so the basis computed by $\mathbf{S B}$ contains 12 elements. The S-pair $\mathcal{S}\left(\phi_{8}, \phi_{4}\right)$ leads to $\phi_{10}$ and it is eliminated by the singular criterion since $\mathfrak{s}\left(x \phi_{7}\right)=\mathfrak{s}\left(\mathcal{S}\left(\phi_{8}, \phi_{4}\right)\right)$ and $x \phi_{7}$ is not regular top $\mathfrak{s}$-reducible. The $S$-pair $\mathcal{S}\left(\phi_{10}, \phi_{1}\right)$ leads to $\phi_{13}$ and it is not considered by $\mathbf{S B}$ since $\phi_{10}$ is not part of $\mathbf{S B}$ 's basis.

\section{F $_{5}$ IS EQUIVALENT TO RB $_{5}$}

The $\mathbf{F}_{\mathbf{5}}$ paper [6] assumes that the input basis elements $g_{1}, \ldots, g_{m}$ are homogeneous. In this section we show that $\mathbf{F}_{\mathbf{5}}$ and $\mathbf{R B}_{\mathbf{5}}$ become the same algorithm with this assumption.

\subsection{The Sig-Poly Pair Optimization}

The notation for $\mathbf{R B}_{\mathbf{5}}$ concerns elements $\alpha \in R^{m}$ while the $\mathbf{F}_{\mathbf{5}}$ paper uses notation based on sig-poly pairs $(\mathfrak{s}(\alpha), \bar{\alpha})$. We believe that the SB notation makes signature computations easier to understand and reason about. However, the cost in time and space for computations on elements of $R^{m}$ is higher than for sig-poly pairs.

Consider that $\mathbf{R B}_{5}$ never performs singular $\mathfrak{s}$-reduction steps, so we never need to know any of the terms in $R^{m}$ other than the leading one - the signature. The singular $\mathfrak{s - r e d u c t i o n ~ s t e p s ~ a p p e a r ~ o n l y ~ i n ~ t h e o r e m s ~ a n d ~ p r o o f s . ~ S o ~}$ it is possible to apply the sig-poly pair optimization when implementing $\mathbf{R B}_{\mathbf{5}}$ (and $\mathbf{R B}$ and $\mathbf{S B}$ ), which involves replacing $\alpha \in R^{m}$ with the sig-poly pair $(\mathfrak{s}(\alpha), \bar{\alpha})$. In $\mathbf{F}_{\mathbf{5}}$ this idea is used in the notation while in $\mathbf{R B}_{5}$ we present it as an optimization. The outcome is the same.

\section{$5.2 \quad F_{5}$ maintains a list of rewriting rules}

The $\mathbf{F}_{\mathbf{5}}$ paper [6] does not contain the phrases "canonical rewriter" and "rewrite order". Instead, there is a pseudo code function named Rewritten that takes a signature as a parameter and returns the canonical rewriter in that signature. The concept of the canonical rewriter is thus represented implicitly in the $\mathbf{F}_{\mathbf{5}}$ paper as that thing which Rewritten returns. We do not specify how the $\mathbf{F}_{\mathbf{5}}$ rewrite order breaks ties since $\mathbf{F}_{\mathbf{5}}$ 's implicit rule for this seems more an arbitrary outcome of how the pseudo code was written than an intentional choice (it is not a function of signature).

In $\mathbf{F}_{\mathbf{5}}$ there is a list of rewriting rules. A rewriting rule is added to the front of the list when an S-polynomial is calculated. When Rewritten is called, it goes through the rewriting rules and returns the first rewriter it finds. The first rewriter found is also the rewriter that was most recently added to the basis. S-polynomials are only calculated for S-pairs whose signature has the current index and the current total degree, so the rewriting rules are added to the front of the list first in order of index of the signature and then in order of total degree of the signature. This proves that $\mathbf{F}_{\mathbf{5}}$ is indeed implicitly using what we have called the $\mathbf{F}_{\mathbf{5}}$ rewrite order.

The only exception to this description is that $\mathbf{F}_{\mathbf{5}}$ does not record $\alpha \in \mathcal{G}$ into the list of rewriters if $\mathfrak{s}(\alpha) \simeq \boldsymbol{e}_{i}$. However, this does not change whether a basis element multiple is rewritable, so this difference does not change the algorithm.

\section{$5.3 \quad F_{5}$ is written to resemble $F_{4}$ reduction}

$\mathbf{F}_{4}$ is an algorithm that speeds up classic polynomial reduction by using a symbolic preprocessing step to turn the reduction of many polynomials into row reduction of a single matrix [4]. The more reductions in the same homogeneous degree that can be done at the same time, the larger the matrix becomes and the more of a speed up there is from using $\mathbf{F}_{4}$ over using classic polynomial reduction. Albrecht and Perry describe a variation of $\mathbf{F}_{5}$ that uses $\mathbf{F}_{4}$ reduction [1].

The pseudo code in the $\mathbf{F}_{\mathbf{5}}$ paper is written without $\mathbf{F}_{\mathbf{4}}$ reduction. However, it is still written in a way that heavily suggests how to adapt the symbolic preprocessing step from $\mathbf{F}_{4}$ to $\mathbf{F}_{\mathbf{5}}$. For example, the $\mathbf{F}_{\mathbf{5}}$ pseudo code is written to process all S-pairs in a homogeneous degree at the same time, which is important when using $\mathbf{F}_{4}$. This is done by having a set $P$ of all S-pairs in the current homogeneous degree. In $\mathbf{F}_{4}$ r-reduction of all the S-pairs in $P$ would then be carried out simultaneously within one big matrix.

The above scheme for $\mathfrak{r}$-reducing all S-pairs in a homogeneous degree does not succeed in getting all of the S-pairs in that homogeneous degree. When reducing an S-pair to a new basis element $\alpha$, it is possible that $\alpha$ can form an S-pair in the same homogeneous degree with another basis element. 
Since $\mathbf{F}_{\mathbf{5}}$ requires the input ideal to be homogeneous, this happens if and only if there is another basis element $\beta$ such that $b \beta$ would top $\mathfrak{r}$-reduce $\alpha$ except that $\mathfrak{s}(b \beta)>\mathfrak{s}(\alpha)$. Call such an S-pair $\mathcal{S}(\beta, \alpha)$ a late $S$-pair. The late S-pairs will not initially be included in $P$ since they only appear after some $\mathfrak{r}$-reductions have already been carried out. This is unfortunate when using $\mathbf{F}_{\mathbf{4}}$ r-reduction because for $\mathbf{F}_{\mathbf{4}}$ $\mathfrak{r}$-reduction we want to identify all of the S-pairs in each homogeneous degree up front - including the late ones.

In $\mathbf{F}_{\mathbf{5}}$ there is a mechanism for discovering late S-pairs while $\mathfrak{r}$-reducing another S-pair. The mechanism is that $\mathbf{F}_{\mathbf{5}}$ allows reducers to increase the signature of the reduction. In that case $\mathbf{F}_{\mathbf{5}}$ will split the $\mathfrak{r}$-reduction into two $\mathfrak{r}$-reductions - one $\mathfrak{r}$-reduction in the old signature that continues to be carried out and another $\mathfrak{r}$-reduction in the new signature that is recorded in $P$. These higher signature $\mathfrak{r}$-reductions are precisely the late S-pairs and this mechanism would allow an $\mathbf{F}_{\mathbf{4}}$ version of $\mathbf{F}_{\mathbf{5}}$ to get all the S-pairs in a degree before doing any $\mathfrak{r}$-reduction. Note that it is possible for such a variant of $\mathbf{F}_{\mathbf{5}}$ to detect more late S-pairs than actually exist because the symbolic preprocessing step does not take account of cancellations.

We have seen that the only effect of $\mathbf{F}_{\mathbf{5}}$ 's higher signature reducers is to indicate how to translate $\mathbf{F}_{5}$ into a variant that uses $\mathbf{F}_{4}$ reduction. The pseudo code for reduction in $\mathbf{F}_{\mathbf{5}}$ looks different from $\mathfrak{r}$-reduction because that pseudo code is not just doing $\mathfrak{r}$-reduction - it is also constructing late $\mathrm{S}$ pairs. This also explains the purpose of the extra conditions on $\mathfrak{r}$-reducers - they are S-pair elimination criteria. So what $\mathbf{F}_{5}$ is doing remains equivalent to $\mathfrak{r}$-reduction, which proves that $\mathbf{F}_{\mathbf{5}}$ is a special case of $\mathbf{R B}_{\mathbf{5}}$ despite $\mathbf{F}_{\mathbf{5}}$ 's highersignature reducers and its multiple results of reduction.

\section{TERMINATION OF RB AND HENCE $F_{5}$}

The proof of termination in the $\mathbf{F}_{\mathbf{5}}$ paper [6] is incorrect. Proving termination of $\mathbf{F}_{\mathbf{5}}$ had been an open problem for a decade before it was settled by Galkin [7]. PHW later proved that F5GEN (and hence RB) terminates [9], which implies that $\mathbf{F}_{\mathbf{5}}$ terminates. Our proof of termination is similar to but significantly simpler than Galkin's and PHW's.

\section{TheOREM 20. The $\mathbf{R B}$ algorithm terminates.}

Proof. Let $\phi_{1}, \phi_{2}, \ldots$ be the sequence of basis elements computed by $\mathbf{R B}$ and let $\mathcal{G}:=\left\{\phi_{1}, \phi_{2}, \ldots\right\}$. RB processes Spairs in increasing order of signature so $\mathfrak{s}\left(\phi_{1}\right)<\mathfrak{s}\left(\phi_{2}\right)<\cdots$. Partition $\mathcal{G}$ into sets $R_{r}:=\left\{\phi_{i} \mid r_{\phi_{i}}=r\right\}$. We prove that there are only finitely many non-empty sets $R_{r}$ and that each $R_{r}$ is finite. Hence $\mathcal{G}$ is finite whereby $\mathbf{R B}$ terminates.

Only finitely many $\boldsymbol{R}_{\boldsymbol{r}}$ are non-empty: If $\alpha \in \mathcal{G}$ then $\mathcal{G}_{\alpha}:=\{\beta \in \mathcal{G} \mid \mathfrak{s}(\beta)<\mathfrak{s}(\alpha)\}$ is a signature Gröbner basis up to signature $\mathfrak{s}(\alpha)$. Call $\alpha \in \mathcal{G}$ minimal if there is no other $\beta \in \mathcal{G}$ such that $\mathfrak{s}(\beta) \mid \mathfrak{s}(\alpha)$ and lt $(\bar{\beta}) \mid$ lt $(\bar{\alpha})$. It follows from Lemma 21 that a non-minimal $\alpha \in \mathcal{G}$ is top $\mathfrak{s}$-reducible by $\mathcal{G}_{\alpha}$. No basis element in $\mathbf{R B}$ is regular top $\mathfrak{s}$-reducible, so if $\alpha \in \mathcal{G}$ is non-minimal, then $\alpha$ must be singular top s-reducible by $\mathcal{G}_{\alpha}$. Thus there exists a $\beta \in \mathcal{G}_{\alpha}$ and a monomial $m \not 11$ such that $\mathfrak{s}(m \beta)=\mathfrak{s}(\alpha)$ and $\operatorname{lt}(\overline{m \beta})=\operatorname{lt}(\bar{\alpha})$ whereby $\alpha$ and $\beta$ lie in the same set $R_{r}$. This shows that there are exactly as many non-empty sets $R_{r}$ as there are minimal basis elements in $\mathcal{G}$. Both $R$ and $R^{m}$ are Noetherian, so there are only finitely many minimal basis elements. Hence there are also only finitely many non-empty sets $R_{r}$.
Each $\boldsymbol{R}_{\boldsymbol{r}}$ is finite: We prove by induction on the finitely many non-empty sets $R_{r}$ that each $R_{r}$ is finite. Assume by induction that all sets $R_{r^{\prime}}$ with $r^{\prime}<r$ are finite. We need to prove that $R_{r}$ is finite. The base case is immediate.

Let $\gamma \in R_{r}$ and let $\mathcal{S}(\alpha, \beta)=a \alpha-b \beta$ be the S-pair that RB regular $\mathfrak{s}$-reduced to get $\gamma$ where $\mathfrak{s}(a \alpha)>\mathfrak{s}(b \beta)$. Let $c$ be the monic greatest common divisor of lt $(\bar{\alpha})$ and lt $(\bar{\beta})$. Then $\frac{1}{c} \operatorname{lt}(\bar{\beta}) \mathfrak{s}(\alpha)=\mathfrak{s}(a \alpha)>\mathfrak{s}(b \beta)=\frac{1}{c} \operatorname{lt}(\bar{\alpha}) \mathfrak{s}(\beta)$ so $r_{\alpha}>r_{\beta}$. Also lt $(\bar{\gamma})<\operatorname{lt}(\overline{a \alpha})$ and $\mathfrak{s}(\gamma)=\mathfrak{s}(a \alpha)$ so

$$
r=\frac{\mathfrak{s}(\gamma)}{\operatorname{lt}(\bar{\gamma})}>\frac{\mathfrak{s}(a \alpha)}{\operatorname{lt}(\overline{a \alpha})}=\frac{\mathfrak{s}(\alpha)}{\operatorname{lt}(\bar{\alpha})}>\frac{\mathfrak{s}(\beta)}{\operatorname{lt}(\bar{\beta})} .
$$

Hence $\alpha \in R_{r^{\prime}}$ and $\beta \in R_{r^{\prime \prime}}$ where $r^{\prime}, r^{\prime \prime}<r$, so $R_{r}$ contains at most as many elements as there are pairs of elements in the set $\cup_{r^{\prime}<r} R_{r^{\prime}}$ which is finite by induction. There may also be one more element with signature $\boldsymbol{e}_{i}$ since those elements do not come from S-pairs. So by induction $R_{r}$ is finite.

Lemma 21 (Eder And Perry [3]). Let $\alpha \in R^{m}$ and let $\mathcal{G}$ be a signature Gröbner basis up to signature $\mathfrak{s}(\alpha)$. If there exists a $\beta \in \mathcal{G}$ such that $\operatorname{lt}(\bar{\beta}) \mid \operatorname{lt}(\bar{\alpha})$ and $\mathfrak{s}(\beta) \mid \mathfrak{s}(\alpha)$ then $\alpha$ is top $\mathfrak{s - r e d u c i b l e ~ b y ~} \mathcal{G}$.

\section{REFERENCES}

[1] Albrecht, M. and Perry, J. F4/5. 2010. http://arxiv.org/abs/1006.4933.

[2] Arri, A. and Perry, J. The F5 Criterion revised. Journal of Symbolic Computation, 46(2):1017-1029, June 2011.

[3] Eder, C. and Perry, J. Signature-based Algorithms to Compute Gröbner Bases. In Proceedings of the 2011 international symposium on Symbolic and algebraic computation, pages 99-106, 2011.

[4] Faugère, J.-C. A new efficient algorithm for computing Gröbner bases (F4). Journal of Pure and Applied Algebra, 139(1-3):61-88, June 1999.

[5] Faugère, J.-C. A new efficient algorithm for computing Gröbner bases without reduction to zero (F5). pages 75-83, 2002.

[6] Faugère, J.-C. A new efficient algorithm for computing Gröbner bases without reduction to zero F5. In Proceedings of the 2002 international symposium on Symbolic and algebraic computation, pages 75-82, July 2002. Revised version from http://fgbrs.lip6.fr/jcf/Publications/index.html.

[7] Galkin, V. Termination of original F5. http://arxiv.org/abs/1203.2402, 2012.

[8] Gao, S., Volny IV, F., and Wang, D. A new algorithm for computing Groebner bases. http://eprint.iacr.org/2010/641, 2010.

[9] Pan, S., Hu, Y., and Wang, B. The Termination of Algorithms for Computing Gröbner Bases. 2012. http://arxiv.org/abs/1202.3524.

[10] Roune, B. H. and Stillman, M. Practical Gröbner basis computation. In Proceedings of the 2011 international symposium on Symbolic and algebraic computation.

[11] Roune, B. H. and Stillman, M. Practical signature Gröbner basis computation. In preparation.

[12] Frank Volny IV. New Algorithms for Computing Gröbner Bases. PhD thesis, Clemson University, 2011. http://etd.lib.clemson.edu/documents/ 1306872881/Volny_clemson_0050D_11180.pdf. 